\title{
Confronting the Models of 3:2 QPOs with the Evidence of Near Extreme Kerr Black Hole
}

\author{
Andrea Kotrlová, Gabriel Török, Eva Šrámková and Zdeněk Stuchlík \\ Institute of Physics, Faculty of Philosophy and Science, Silesian University in Opava, \\ Bezručovo nám. 13, CZ-74601 Opava, Czech Republic \\ email: andrea.kotrlova@fpf.slu.cz
}

\begin{abstract}
The black hole mass and spin estimates assuming various specific models of the $3: 2$ high frequency quasi-periodic oscillations (HF QPOs) have been carried out in Török et al. (2005, 2011). Here we briefly summarize some current points. Spectral fitting of the spin $a \equiv c J / G M^{2}$ in the microquasar GRS 1915+105 reveals that this system can contain a near extreme rotating black hole (e.g., McClintock et al., 2011). Confirming the high value of the spin would have significant consequences for the theory of the HF QPOs. The estimate of $a>0.9$ is almost inconsistent with the relativistic precession (RP), tidal disruption (TD), and the warped disc (WD) model. The epicyclic resonance (Ep) and discoseismic models assuming the c- and gmodes are instead favoured. However, consideration of all three microquasars that display the $3: 2$ HF QPOs leads to a serious puzzle because the differences in the individual spins, such as $a=0.9$ compared to $a=0.7$, represent a generic problem almost for any unified orbital $3: 2$ QPO model.
\end{abstract}

Keywords. Accretion, accretion disks; X-rays: binaries; black hole physics

\section{The spin implied by individual models}

Assuming the Kerr geometry, the Keplerian and epicyclic orbital frequencies $\left(\nu_{K}, \nu_{\mathrm{r}}\right.$ and $\nu_{\theta}$ ) for a given radius depend only on mass $M$ and spin $a$ of the black hole. It is therefore possible to infer the black hole spin or mass from the observed $3: 2$ frequencies and concrete orbital QPO models.

The 3:2 QPO frequencies in GRS 1915+105 are given by

$$
\nu_{U}=168 \pm 3 \mathrm{~Hz}, \quad \nu_{L}=113 \pm 5 \mathrm{~Hz} .
$$

Assuming relation (1.1) and the well known formulae for the orbital frequencies, we calculate the implied mass-spin functions for the models associating the $3: 2$ QPOs with common radii by means of the definition relations given in Table 1. Following Török et al. (2005) and taking into account the estimated range of the mass of GRS 1915+105,

$$
10 \mathrm{M}_{\odot} \leqslant M \leqslant 18 \mathrm{M}_{\odot}
$$

we infer the expected ranges of the spin. The results are presented in Table 1.

The above considered models assume that both of the observed $3: 2$ frequencies are produced by the same mechanism and excited at a certain (common) preferred radius. For the discoseismic modes the individual observed QPOs correspond to different modes located at different radii. The frequencies of these modes depend on the black hole spin and the speed of sound in the accreted gas, and scale as $1 / M$. The mass ranges implied by combinations of the fundamental discoseismic modes overlap with those observationally determined only for the model relating the 3:2 QPOs to the c-mode (corrugation 
Table 1. Frequency relations corresponding to individual QPO models examined by Török et al. (2011) and the resulting ranges of spin implied by the $3: 2$ QPOs in GRS $1915+105$.

\begin{tabular}{|l|l|l|c|c|}
\hline Model & \multicolumn{1}{|c|}{ Frequency relations } & $\nu_{\mathbf{K}} / \nu_{\mathrm{r}}$ or ${ }^{*} \nu_{\theta} / \nu_{\mathrm{r}}$ & $\mathbf{a} \sim \mid$ \\
\hline RP & $\nu_{L}=\nu_{K}-\nu_{\mathrm{r}}$ & $\nu_{U}=\nu_{K}$ & $3 / 1$ & $<0.55$ \\
TD & $\nu_{L}=\nu_{K}$ & $\nu_{U}=\nu_{K}+\nu_{\mathrm{r}}$ & $2 / 1$ & $<0.45$ \\
WD & $\nu_{L}=2\left(\nu_{K}-\nu_{\mathrm{r}}\right)$ & $\nu_{U}=2 \nu_{K}-\nu_{\mathrm{r}}$ & $2 / 1$ & $<0.45$ \\
Ep & $\nu_{L}=\nu_{\mathrm{r}}$ & $\nu_{U}=\nu_{\theta}$ & $3 / 2^{*}$ & $0.65-1$ \\
Kep & $\nu_{L}=\nu_{\mathrm{r}}$ & $\nu_{U}=\nu_{K}$ & $3 / 2$ & $0.70-1$ \\
RP1 & $\nu_{L}=\nu_{K}-\nu_{\mathrm{r}}$ & $\nu_{U}=\nu_{\theta}$ & - & $<0.80$ \\
RP2 & $\nu_{L}=\nu_{K}-\nu_{\mathrm{r}}$ & $\nu_{U}=2 \nu_{K}-\nu_{\theta}$ & - & $<0.45$ \\
\hline
\end{tabular}

Note: The middle column indicates the ratio of the epicyclic frequencies determining the radii corresponding to the observed $3: 2$ ratio. The indicated ranges of spin also represent total spin ranges for the whole group of the three microquasars.

vertically incompressible waves near the inner edge of the disk) and g-mode (inertialgravity waves that occur at the radius where $\nu_{\mathrm{r}}$ reaches its maximum value) provided that $0.90 \leqslant a \leqslant 0.94$. Details and references are given in Török et al. (2011).

\section{Conclusions}

The internal (epicyclic) resonance and the discoseismic model (dealing with c- and gmodes) are favoured in the case of GRS $1915+105$ provided that $a>0.9$. On the contrary, the TD, WD, RP, and RP2 models are disfavoured. This statement was inferred assuming that $\nu_{\mathrm{K}}, \nu_{r}$, and $\nu_{\theta}$ were the exact geodesic frequencies. Analysis including the influence of non-geodesic effects would require a very detailed study. A rough estimate of their possible relevance can be done assuming the relative non-geodesic correction $\Delta \nu$ (Török et al., 2011), which is needed to match the observations of GRS $1915+105$ with a given model for a certain spin. For $a \in(0.9,1)$ and the RP model, it changes from $-40 \%$ to $-60 \%$. The same is roughly true for the TD and WD models, while for the RP2 model the required correction is even higher. Thus, the above result is justified, except when very large non-geodesic corrections are taken into account. Only the RP1 model can survive with corrections of $|\Delta \nu|$ up to $\sim 20 \%$, but the present physical interpretation of this model is unclear (see Török et al., 2011 for references).

Török et al. (2005) pointed out that since the 3:2 QPO frequencies in microquasars scale roughly as $\nu_{\mathrm{U}} \doteq 2.8\left(M / \mathrm{M}_{\odot}\right)^{-1} \mathrm{kHz}$, their spins implied by a given resonance model should not much vary among them. If very different spins in GRS 1915+105, GRO J1655-40 and XTE J1550-564 were confirmed, the difficulty of matching all the observed 3:2 frequencies would clearly be rather generic for most of the orbital QPO models.

Because of the generic $1 / M$ scaling, the above difficulty also arises for a unified $3: 2$ QPO model assuming fundamental discoseismic modes.

\subsection{Acknowledgements}

The authors acknowledge the research grant GACR 209/12/P740 and the project CZ.1.07/2.3.00/20.0071 - "Synergy" supporting international collaboration of the Institute of Physics at SU Opava.

\section{References}

McClintock, J. E., Narayan, R. Davis, S. W., et al. 2011, CQG, 28, 114009

Török, G., Abramowicz, M. A., Kluźniak, W., \& Stuchlík, Z. 2005, A\&ऽA, 436, 1

Török, G., Kotrlová, A., Šrámková, E., \& Stuchlík, Z. 2011, A\&̈A, 531, 59 